\title{
Strategic use of statistical thinking in drug development
}

\author{
Frank W. Rockhold* \\ SmithKline Beecham Pharmaceuticals, Research and Development, 1250 South Collegeville Road, P.O. Box 5089, \\ Collegeville PA 19426-0989, U.S.A.
}

\begin{abstract}
SUMMARY
The role of the statistician and statistical thinking in the pharmaceutical industry has evolved greatly in the last four or five decades. Regulatory developments and the changing face of the science of drug development have driven this evolution. The increasing regulatory requirement for statistical input in critical areas has facilitated a wider range of applications. The pace of change of science in general has brought statisticians into contact with a wider range of potential customers. More importantly, it has allowed the statistician to become increasingly involved in strategic issues with the possibility of influencing the direction of the business. However, it is not clear that the statistical profession in industry is adequately prepared for these opportunities either in attitude or training. Changing the statisticians' approach to their role and acquiring the correct training and experience are critical for the profession to optimize their contribution to the drug discovery and development processes. Copyright (c) 2000 John Wiley \& Sons, Ltd.
\end{abstract}

\section{INTRODUCTION}

This paper discusses what I refer to as a strategic use of statistical thinking in drug development (and discovery), which is the direction in which I think the statistics profession in industry should continue to move over the next decade. The first section describes the evolution of the use of statistics in the pharmaceutical industry. The second section lists some of the things that have driven this change. The third section reviews current challenges faced with the statisticians' current research collaborators, and the last two sections consider the future evolution of the statisticians' role as well as the impact on statistical education. You will note that in this paper I distinguish between 'statistician' and 'statistical thinking', as I believe if the former do their job properly then the latter will be done far more often by many non-statisticians.

\section{EVOLUTION OF STATISTICS IN DRUG DEVELOPMENT AND DISCOVERY}

How has our role evolved? Figure 1 gives a schematic of recent history. Interestingly, this tracks closely with the evolution of clinical research [1]. There were some groundbreaking folks back in

\footnotetext{
*Correspondence to: Frank W. Rockhold, SmithKline Beecham Pharmaceuticals, Research and Development, 1250 South Collegeville Road, P. O. Box 5089, Collegeville PA 19426-0989, U.S.A.
} 


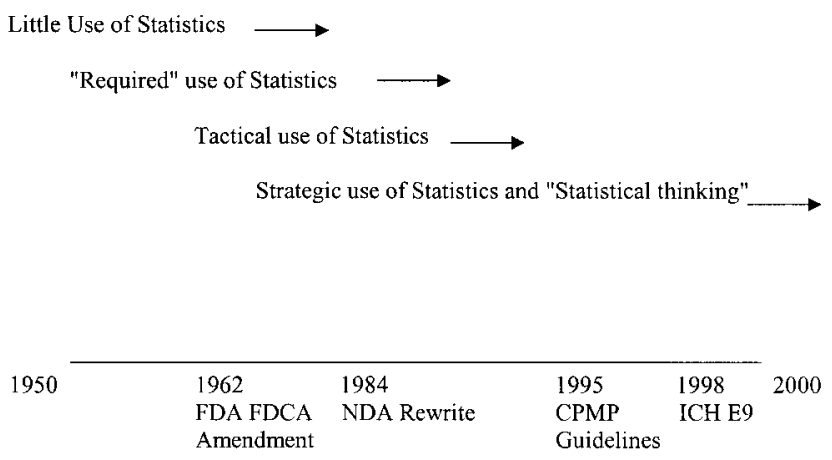

Figure 1. How has the use of statistics in drug development evolved over time?

the mid- to late-1950s who began using statistics routinely in the pharmaceutical industry and in limited areas. However, industry did not move to 'required' use of statistics and (where 'required' is obviously in the eyes of the beholder) until the 1960s and then for the most part in areas where it was required for regulatory and publication reasons. We then moved into a phase (where some might argue we still are) of the tactical use of statistics. Here applications went beyond what was absolutely required, but were still directed at applying methodology to traditional problems. Today expansion has occurred and more time is spent on the strategic use of statistical thinking. The statistician brings a different thought process to the table in research and business discussions and now this is increasingly being recognized as a valuable perspective for research and business efforts. Statisticians themselves have changed in line with the need to address this evolution. This discussion is in line with a paper by Porter who addresses a small window of this time period in detail [2].

In the pharmaceutical industry environment (given the background of the statisticians and the customers), how has statistics as a profession and the statistician's role in particular evolved? Back in the mid-1960s and early-1970s the strategy was hire some statisticians to aid in gaining marketing approval from the U.S. Food and Drug Administration (FDA). The amendments in 1962 to the Food, Drug, and Cosmetic Act required proof of efficacy. That was often referred to as the 'statisticians' lifetime employment act', because the industry started hiring statisticians because they realized the FDA was going to require statistically valid results and the FDA was hiring statisticians themselves. This trend increased even more sharply when the New Drug Application Rewrite Guidelines were released in the early-1980s which required not only an FDA statistical review on all new product applications, but that the industry statistician be a co-author on clinical trial reports. However, the industry statisticians were still largely viewed as 'number crunchers'; just get the analyses done so they could be agreed with FDA and other regulatory agencies. The focus at the time was primarily on clinical trials and some manufacturing issues. This focus gradually shifted to earlier phases of the research process, but even so still largely only involved 'blessing' clinical trial designs and sample sizes without the statistician being one of the key drivers in the design itself. There was very little input outside of what was deemed by the customer as necessary and fairly low involvement in non-clinical areas to any extent at all.

How far have we come in the last 30 years? Statisticians are now viewed as absolutely critical for efficient and effective drug development. What has helped the statistician have a larger contribution 
is that most people are now researching and developing products with the end in mind. This focus on the process of discovery and development should (and has) put more emphasis on design of experiments and programmes in the non-clinical as well as the clinical arena. Thus, the statistician is crucial in leading researchers through the logical steps in the development process. The statistician is now involved in the full range of pharmaceutical research activities: discovery; non-clinical and clinical development; health economics; portfolio management, and manufacturing. More importantly, they participate in the strategy for development, design of the development plan itself, consult on decision criteria and risk management. The last two have been recognized for some time [3] as a critical area for involvement of statisticians. To do this means we must move away from operational activities. In many cases, basic scientists in discovery and non-clinical development are performing their own routine analyses, and allow statisticians to focus on the more complicated analyses. Industry is slowly moving in that direction in the area of clinical trials.

To facilitate this transition, there has been much wider use of computer application programmers as a professional group to support statisticians in the past 20 years. This has done two things. First, it has freed up the statisticians trying to focus more on the statistical part of the problem and second has actually resulted in more efficient and better validated software that has been more readily acceptable to regulatory agencies.

\section{WHAT HAS STIMULATED THIS CHANGE?}

There are a number of key factors that are part of the overall impetus of the changes described in the previous section. These can be grouped in the following broad categories:

(i) the scope and nature of problems have changed;

(ii) statistical 'champions' have taken on new and higher profile roles and helped changed the overall landscape of the profession in industry;

(iii) the recognition in research and development that information is the key commodity and product of the research process;

(iv) customers have become much more educated and demanding about the use of statistics as applied to their problems.

(v) statisticians have realized that the teaching role should not be left at the university and that this is critical in promoting the use of statistical thinking in industry;

(vi) regulatory changes have given the profession both more visibility in the drug development process and increased the number and complexity of problems.

Some of these changes in substance or attitude have been substantial. At the end of the day we expend a great deal of resources to develop a new medicine in research and development and what we have at the end is information. This realization has been a huge boost to the importance of the role of statistics in industrial research. A lot of key reforms have taken place in the regulatory arena, particularly with the International Conference on Harmonization, or ICH, which have put the statistician in much more of a position of leverage and authority in drug development. This is most clearly characterized in the publication of ICH E9: Statistical Principles for Clinical Trials [4].

In the next section, the scope of challenges faced through the collaborators of the statistician in industry are reviewed. This has been one of the biggest agents for change in recent years. 


\section{CUSTOMERS AND RESEARCH PARTNERS: CURRENT CHALLENGES}

As noted above, many of the new challenges and advances in statistical methods and thinking have been created or inspired by our research collaborators. Who are these customers and collaborators? In our business it is ultimately the patients and the physicians who are using our products. In the shorter term, it is $\mathrm{R}$ and $\mathrm{D}$ scientists, the regulators who pass judgement over our submissions, and journal editors who deal with publications on research that either the statistician themselves are doing or more specifically that our collaborators are performing. The management of the company and the stockholders of the company have very different needs and interests, as do healthcare providers and purchasers. However they all have one thing in common; they all have a need for speed, quality and low cost.

Research and development scientists clearly have their own views and needs and they want it done fast. In reality they need it done the 'right' way. Statisticians are the guardians of many of the 'right' ways and they bring a unique set of skills to the table. Since their way is not always the only 'right' way (there is as much art as there is science in what statisticians do), is it important for the statistician to have the ability to state their case. Healy [5] and others have noted that statisticians actually thrive in the team environment. Statistics in this environment can be encapsulated as the implementation and preserving of the scientific method, but collaboration and education of the customers are really the key to the identification and solution of the specific problems.

Statistical thinking in the discovery and development process is becoming more widespread. In the discovery arena, identifying targets using molecular genetics, identifying compound leads using combinatorial chemistry and high through-put screening, are all greatly enhanced by experimental design, as is the confirmation of compound activity done through application of design in non-clinical and clinical experimentation. Understanding the full value of the asset through portfolio modelling and post-marking safety or surveillance, and, once the product is on the market, understanding where new opportunities exist for the use of existing products, are yet a few of the new and challenging opportunities open to statisticians. One has only to observe the increased number of statisticians taking on senior management roles in industry to be convinced that the role of statistics is gaining ground and will continue to do so into the future. This will be discussed further in the next section.

Many technology improvements have changed drug discovery and development. Numerous advances have been seen in high throughput screening and other advanced compound screening technologies. These require automated experimentation that in turn requires automated statistical analysis and decision criteria, as humans cannot process decisions when thousands and thousands of compounds are being screened every day. In all areas we need decision rules that are really driven by statistical methodology and thinking. We collect increasingly large amounts of data (and maybe information). Data mining technologies, which statisticians rightfully recognize as old statistical methods being put to new uses, are becoming more of an integral part of what we do day-to-day. The statistician must also learn to leverage the technology changes such as high end computing and advanced information technologies used in data capture and analysis systems. This will enhance both the tactical (by reducing the need for some operational activities) and strategic dimension of their job.

Government regulators are charged with protecting the public health and while they are also interested in fundamental science, they also insist on a clear research history and audit trails. While they are interested in all phases of clinical trial design, they have a keen interest in type I error 
rates when it comes to judging efficacy to avoid letting ineffective treatments on the market. At the end of the day they are always concerned about benefit-to-risk ratio and increasingly have advocated formal analyses of safety as well. Many of these concerns also exhibit themselves as new or altered regulatory guidelines. These may differ by disease area and/or geographic region, thus adding to the complexity of the statistician's job.

What does the management in the company desire from statisticians? Most importantly they certainly want useful information to make product decisions. They seek a balance between business, basic science, clinical and regulatory science. They need clear risk management and decision criteria and this is one area where statisticians are beginning to have a clear impact, but there is major room for improvement. To the company, type I and type II error control are both important, but especially type II error. One does not want to invest hundreds of millions of pounds into a programme only to lack the sensitivity to detect the outcome of interest. The statistician must continue to educate people about these risks and how to estimate and manage them and be a key partner in creating efficient and effective development strategies. Management wants to get to the right answer for the compounds they have in the portfolio with minimal risk of failure. The shareholders want to return on their investment, which also involves a risk management process. In successful companies the risks identified by the shareholders, senior management and the scientists are in alignment and thus make for a much more efficient and effective research process.

Increasingly we have demands from payers and formularies, healthcare systems, national governments etc. They want comparability data in monetary terms. In other words, how much does it cost to use this therapy versus what already exists? Then they demand strict control over so-called 'type III' errors: the probability of using a drug that is not cost effective or cost beneficial.

While we have done well as a profession evolving in line with our customers, we as a profession have not always been the most aggressive at seeking new areas to apply our talents and to be truly successful we must actually work to drive to change the horizon ourselves. This is discussed in the next section.

\section{FUTURE EVOLUTION OF THE STATISTICIAN'S ROLE}

As discussed in the previous sections, the role of the statistician has evolved greatly. Statisticians have on the whole adapted to change well, but as the pace of change increases will the profession be able to continue to adapt or do we need to rethink our strategy? I believe we are going to continue to expand the role and transfer from what is today largely a tactical role to one of more of a consistently strategic role and streamline the operational day-to-day role. Both of these need to happen in parallel for the profession to be successful.

Statistics as a profession and the statistician's importance will continue to increase because the need for efficiency and speed will not go away. We are under increasing pressure to go faster and faster, but at the same time not sacrifice quality. We have made enormous strides in the preclinical area in experimental design and we need to further expand our impact in the clinical arena. In addition, there is a need for precise and quantifiable decision making process and risk assessment. In order to go fast, but understand and minimize the risks of our decisions, we need to have a very specific decision criteria in our development plans and experiments.

Continuing to educate colleagues on the concepts of variability, uncertainty and risk is critical, and as statisticians are increasingly taking on more and more senior roles in companies, they now 
Table I. Impact on statistical education.

Increasing emphasis on experimental design

Continued emphasis on communication/presentation skills

General science

Decision theory

Risk assessment

are in a position to continue this education at high levels. The impact of statistics will fall short of the mark if the other people at the table do not understand the language/tools we are trying to employ. Once again, that information will be the capital or the currency in research means the statistician is well positioned to succeed.

\section{IMPACT ON EDUCATION AND CAREER DEVELOPMENT OF STATISTICIANS}

As we move into these new arenas we need to ask ourselves: do we have the right career development strategies in place for statisticians to achieve what has been discussed in the paper? This relates not only to pharmaceutical industry statisticians but also to those in the academic and government environment. Are we providing statisticians with the right background to succeed? As we move from the tactical to strategic applications, are we ready? Are statisticians as a class trained to jump in when the time is right? I have many examples in my career experience where the fact that statistics fail to be applied at a given time in a drug development process was not the fault of the customers or collaborators but the statisticians themselves for failing to assert themselves when the time was right. This in the face of the fact they recognized the need to do so! This reflects what Bross [6] notes as 'anyone who acts like a shoe clerk will end up being treated like a shoe clerk', and Healy [7] states the need for the statistician to 'look over the fence'. If we hope to make these leaps in the application of statistics in these areas we need to understand and really ask ourselves seriously, do we have the right kind of people and have we trained them appropriately?' Are we and should we be turning statisticians into business leaders if we are going to take seriously some of the applications I indicated earlier? Do we need to stress that our value comes from focusing on the scientific and business solutions and not strictly statistical methodology? Sending statisticians to work in other places in our companies to help spread statistical thinking takes courage on the statistician's part, but will pay huge dividends. Are we using the right education, pedigree, and for what jobs? We need to make sure we have created the correct career ladders and progression so we can then create the appropriate recruiting strategy.

This all has an impact on the way we train and develop statisticians. Table I outlines the key aspects that need to be part of the future statisticians' education in order to succeed in industry. Some of these are a routine part of core graduate programmes, but the emphasis on general scientific and communication skills must increase.

In conclusion, the statisticians in industry in the future need to consider being successful in multiple career tracks and move outside their comfort zone and have a solid balance between process and technical skills. Technical skills are necessary but not sufficient. All this will allow the statistician in our industry to be better prepared and give the ability to continue to increase their level of influence in the research process. 


\section{ACKNOWLEDGEMENTS}

I would like to acknowledge Dr Greg Enas, as I have benefited greatly from discussions with him over the years on this and many topics. In addition, I would like to thank the reviewers who made the quality of this paper substantially better through their comments.

\section{REFERENCES}

1. Parascandola M. The history of clinical research in the United States. Journal of Clinical Research Practice 1999; 1:7-20.

2. Porter MA. The role of the statistician in industry. Statistician $1993 ; \mathbf{4 2}: 217-227$.

3. Healy MJR. New methodology in clinical trials. Biometrics 1978; 34:709-712.

4. ICH E9 Expert Working Group. ICH Harmonised Tripartite Guideline: Statistical Principles for Clinical Trials. Statistics in Medicine 1999; 18:1905-1942.

5. Healy MJR. The varieties of statistician. Journal of the Royal Statistical Society, Series A 1973; 136:71-74.

6. Bross IDJ. The role of the statistician: scientist or shoe clerk. American Statistician 1974; 28:126-127.

7. Healy MJR. Prospects for the future. Where has statistics failed? Journal of the Royal Statistical Society, Series A 1984; 147:368-374. 